\title{
Error Analysis of Magnetohydrodynamics Angular Rate Sensor under the Influence of a Non-uniform Magnetic Field
}

\author{
Yue $\mathrm{Ji}^{1,}$, Xingfei $\mathrm{Li}^{1, \mathrm{~b}}$ and Tengfei $\mathrm{Wu}^{1, \mathrm{c}}$ \\ 1 State Key Laboratory of Precision Measuring Technology and Instruments, Tianjin University, \\ Tianjin 300072, China; \\ ajiyue@tju.edu.cn, bIxtju@hotmail.com, cwtf@tju.edu.cn
}

Keywords: Angular rate sensor, Magnetohydrodynamics (MHD), Non-uniform magnetic Field

\begin{abstract}
In order to improve the dynamic measurement capability of magnetohydrodynamics (MHD) angular rate sensor (ARS) furtherly, this paper gives the error analysis of MHD ARS under the influence of a non-uniform magnetic field. In this work, the numerical simulation using 3D-Magnetostatic and FLUNET coupled with User Defied Function (UDF) is made. It is found that the inhomogeneity effect on the velocity fluctuations is not outstanding, whereas the effect on the electric potential is considerable. Sinusoidal curve fitting method is introduced to characterize the sensor output errors. The results indicate that the random error of the sensor output may be generated by the magnetic field inhomogeneity especially at the peak and valley of angular vibration. This finding can provide a reference for the optimization of the sensor design.
\end{abstract}

\section{Introduction}

The magnetohydrodynamic (MHD) angular rate sensor (ARS) has been developed and demonstrated in a range of applications [1] for no mechanically moving parts and low power consumption. The sensor with ultralow noise is capable of monitoring angular vibrations of acquisition, tracking, and pointing support structures used in space experiments over thousand hertz range of frequency. [2] For impact resistance and low cost, the MHD ARS is also suitable for making accurate measurements of the inertial motions of head kinematics test [3], high-g sled and other automotive safety researches[4]. In order to improve the dynamic measurement capability furtherly, some evaluations of the MHD ARS have been studied. Martin et al.[5] showed that the head angular accelerator in impact testing could be obtained by the differential of MHD ARS but the results had poor consistency. Merkle et al.[6] indicated that the MHD ARS had large discrepancies from the benchmark in determining the angular displacement and attributed the problem to the scale factor drift. These tests necessitate the sensor's modeling and error analysis. Pinney et al.[7] showed the coherence function method to give the noise floor of the sensor for predicting the angular position error. Laughlin et al. [8] described the tested frequency response curves and proposed a simplified model of MHD ARS, which ignored some error sources in theory. Xu et al.[9] presented the numerical simulation results and draw the conclusion that the sensor output could not be calculated at a single magnetic field value, but the effect of the non-uniform magnetic field on the measurement was not given. Many researches have shown that the fringing magnetic field has influence on the MHD characteristics[10] . This paper will perform the study to reveal the details of the flow fields or electromagnetic field under the influence of a non-uniform magnetic field in MHD ARS.

\section{Modeling of MHD ARS}

Working principle The basic principle of the MHD ARS is the electromagnetic induction phenomenon of conductive fluid. As shown in Fig.1, the upper ring is filled with conductive fluid and a permanent magnet is fixed with the case. The top and bottom sides are parallel insulating plates with height $h$, whereas inside and outside walls are concentric conducting cylinders with radii $r_{i}$ and $r_{o}$. When the case installed with a measured object rotating with an angular velocity $\Omega$, the fluid remains 
relatively stationary with respect to the inertial frame due to inertia and mobility. Then the fluid moves through the rotating magnetic flux $\mathbf{B}$ and a radial electromotive force $\varphi$ is generated.

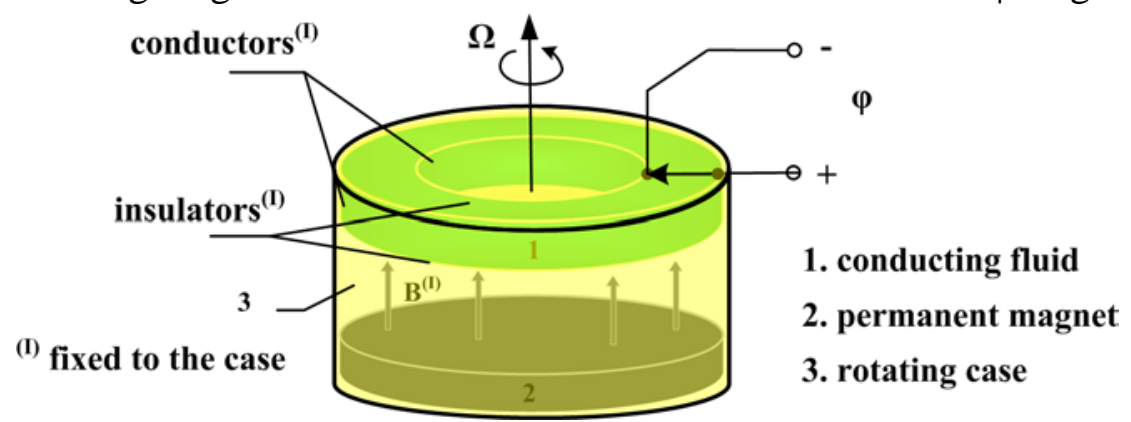

Fig.1 Schematic diagram of MHD ARS's principle

Ignoring the secondary flow and induced magnetic field, the relation between the output and angular velocity can be expressed as equation (1) [8]:

$$
\frac{\varphi(s)}{\Omega(s)}=\frac{B_{0} l R s}{s+\sigma B_{0}^{2} / \rho+v / h^{2}}=\frac{B_{0} l R s}{s+v / h^{2}\left(1+M^{2}\right)}
$$

Where $l$ is the width of the channel, $R$ is the equivalent radius and can be obtained by $R=\left(r_{i}+r_{o}\right) / 2$ and $M$ is Hartmann number, which means the ratio of electromagnetic force and viscous force.

Governing Equations The motion of the conductive fluid and electromagnetic field in the MHD ARS is governed by the following equations in non-inertial system:

$$
\begin{aligned}
& \frac{\partial \mathbf{u}}{\partial t}+(\mathbf{u} \cdot \nabla) \mathbf{u}=-\nabla \mathbf{p}+v \cdot \nabla^{2} \mathbf{u}+\mathbf{j} \times \mathbf{B}-\frac{\partial \boldsymbol{\Omega}}{\partial t} \times \mathbf{r}-2 \mathbf{\Omega} \times \mathbf{u} \\
& \nabla \cdot \mathbf{u}=0 \\
& \frac{\partial \mathbf{B}}{\partial t}=\nabla \times(\mathbf{u} \times \mathbf{B})+\frac{1}{\mu \sigma} \nabla^{2} \mathbf{B} \\
& \nabla \cdot \mathbf{B}=0
\end{aligned}
$$

Where $\sigma$ is electrical conductivity, $\rho$ is mass density, $v$ is kinematic viscosity coefficient and $\mu$ is magneto conductivity. The total magnetic field, the electric current density, the pressure field and the relative velocity field are defined by $\mathbf{B}, \mathbf{j}, \mathbf{p}$ and $\mathbf{u}$ respectively.

\section{Simulation}

The magnitude of the magnetic field $B$ in the conducting field in the annulus is obtained using 3D-Magnetostatic, which is shown in Fig.2 (a). The inside and outside radii are respectively $4 \mathrm{~mm}$ and $16 \mathrm{~mm}$. The height of the fluid ring is $4 \mathrm{~mm}$. It can be seen that the magnitude at the bottom is larger than at the top. The magnitude along the radial direction descends from the inside to the outside. These results can be attributed to the different distances from the permanent magnet and the inhomogeneity of magnet. The average value of the magnitude is $0.18 \mathrm{~T}$ and the inhomogeneity of the magnetic field $B_{\gamma}$ is about $72.5 \%$ calculated according to the equation (6).

$$
B_{\gamma}=\left(B_{\max }-B_{\min }\right) / B_{\text {average }}
$$

The numerical simulation of the fluid motion is employed using FLUENT coupled with the User Defied Function (UDF). The MHD ARS is always required to sense angular vibration, so the angular velocity in the simulation is chosen at $\Omega=\sin (2 \pi \cdot f \cdot t)(f=10 \mathrm{~Hz})$. The simulation under the non-uniform magnetic field shown in Fig.2 (a) is made compared with under the magnetic field at the average value $B=B_{\text {average }}=0.18 \mathrm{~T}$. The maximum velocity at different radii from $r_{i}$ to $r_{o}$ in the two situations are compared in the Fig.2 (b). It can be found that the influence of the magnetic field inhomogeneity on the velocity fluctuations is not significant. However, the measurement of MHD ARS mainly depends on the generated voltage. The electrical potential at the plane $\theta=0$ in the two situations are presented in Fig.3. The sharp contours shown indicates that the electrical potential is dramatically influenced by the magnetic field especially at $t=1 / 4 \mathrm{~T}$ and $\mathrm{t}=3 / 4 \mathrm{~T}$. At the same radius, the electrical 
potential at the bottom is larger than at the top.

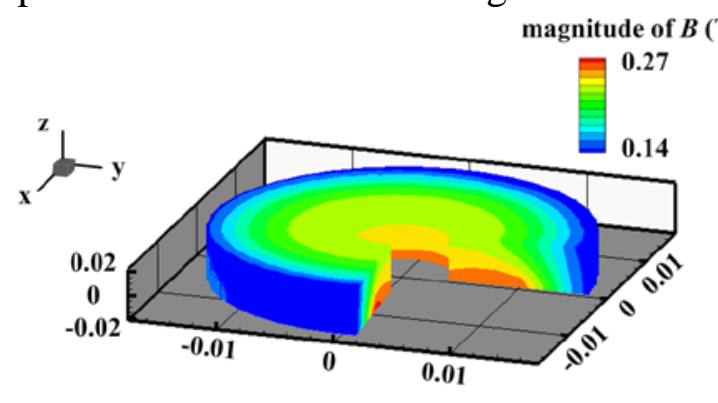

(a)

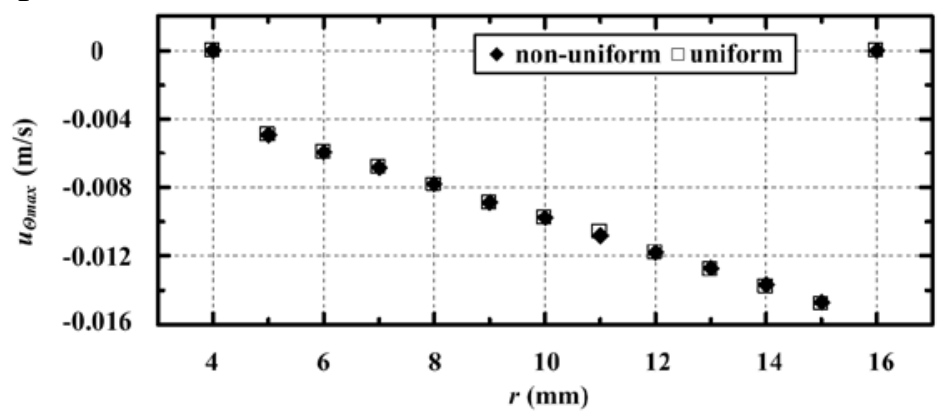

(b)

Fig.2 (a) Magnitude of the magnetic field B in the conducting fluid in the annulus.

(b) Simulation results of the velocity along the radial direction in conducting fluid in the annulus respectively under non-uniform and uniform magnetic field

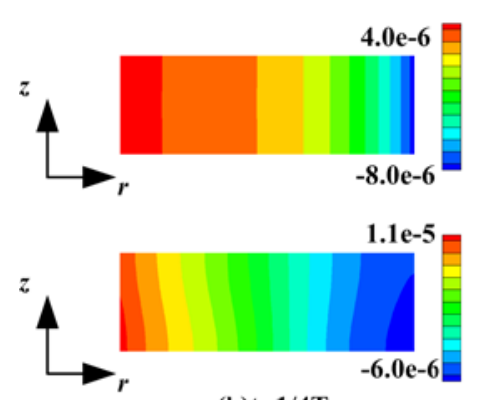

(b)t=1/4T
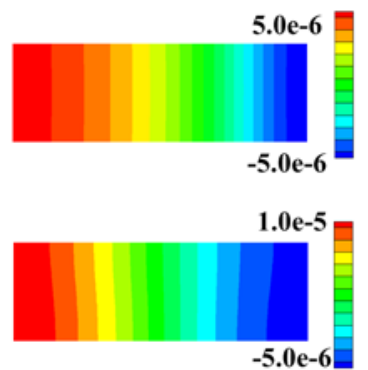

(c) $\mathrm{t}=1 / 2 \mathrm{~T}$

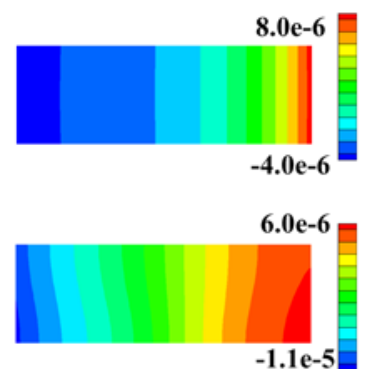

(d) $\mathbf{t}=\mathbf{3} / \mathbf{4} \mathrm{T}$
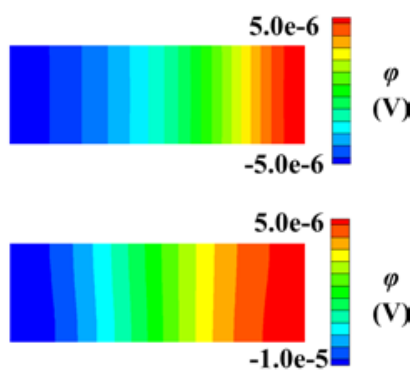

(d) $\mathbf{t}=\mathbf{T}$

Fig. 3 Contours of electric potential at the moment $t=1 / 4 \mathrm{~T}, 1 / 2 \mathrm{~T}, 3 / 4 \mathrm{~T}$ and $\mathrm{T}$

under uniform and non-uniform magnetic field

The output voltage in the two situations between the inside and outside electrodes is illustrated in Fig. 4. The fitting curves of the wave function $f(x)=A \sin (\omega x+\Phi)$ are obtained according to the simulation results. The R-square of fitting results under the uniform field is 0.9958 , while it under the non-uniform magnetic field is 0.9763 . By contrast, we can find that the error generated by the magnetic field inhomogeneity mainly occurs in at the peak and valley moment, which can also be confirmed in Fig.3.

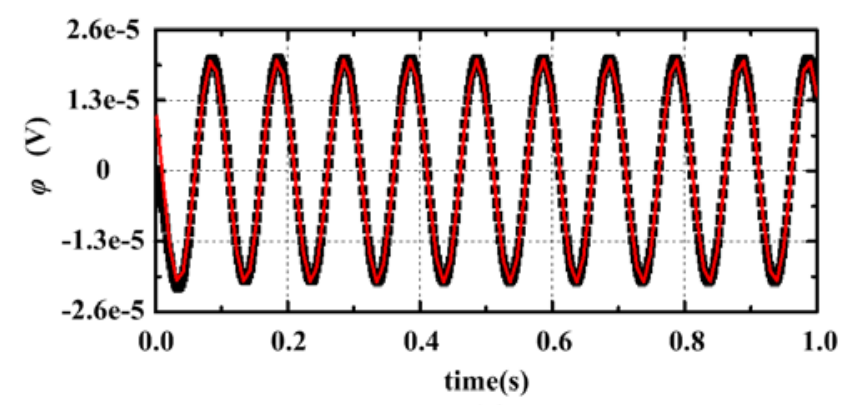

(a) uniform

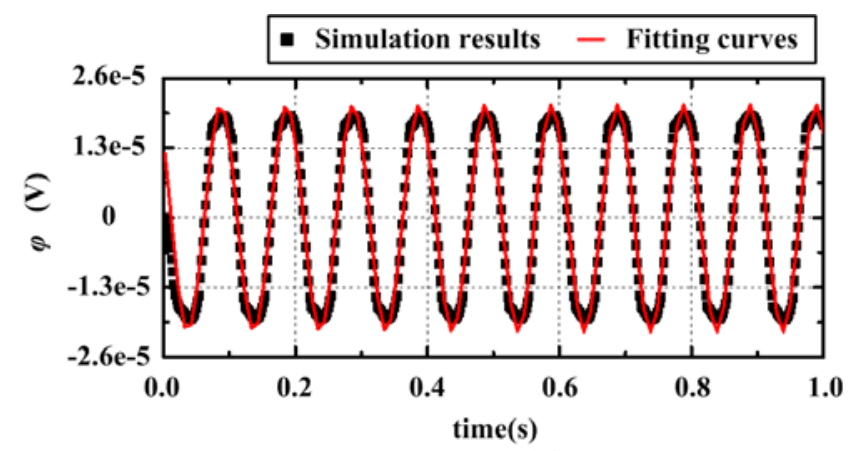

(b)non-uniform

Fig. 4 The generated voltage under uniform and non-uniform magnetic field

Table1 .The R-square of the fitting curves at different inhomogeneity of magnetic field

\begin{tabular}{cc}
\hline inhomogeneity of magnetic field & R-square \\
\hline $72.5 \%$ & 0.9763 \\
$54.8 \%$ & 0.9801 \\
$37.2 \%$ & 0.9865 \\
$19.4 \%$ & 0.9906 \\
uniformity & 0.9958 \\
\hline
\end{tabular}

To reduce the inhomogeneity of magnetic field, the magnetic circuit is adjusted by changing the height of the fluid ring and adding some magnetic material. To verify the effect furtherly, the R-square of the fitting curves at different inhomogeneity of magnetic field are presented in Tab.1, 
when rotating at $\Omega=\sin (2 \pi \cdot f \cdot t)(f=10 \mathrm{~Hz})$ in simulation. It is apparent that the R-square increases as the inhomogeneity decreases. We can draw the conclusion that the inhomogeneity of magnetic field may generate the random error in the MHD ARS measurement, which should be paid full attention.

\section{Summary}

We have investigated the influence of the non-uniform magnetic field on the MHD ARS by the modeling and numerical simulation. The inhomogeneity effect on the velocity fluctuations is found to be not outstanding while the effect on the electric potential is considerable. The output fluctuations can be evaluated by the R-square of fitting curves. The random error may be generated by the magnetic field inhomogeneity especially at the peak and valley of angular vibration. The design of magnetic circuit in the MHD ARS should be optimized in the sensor to give the quantization error analysis in the actual experiment.

\section{Acknowledgments}

This work is supported by the Foundation of the National Natural Science Foundation of China (No. 61304243 and 61427810).

\section{References}

[1] M. E. Pittelkau, Sensors for attitude determination. Encyclopedia of Aerospace Engineering, Wiley, Chichester 2010.

[2] H. R. Sebesta, J. E. Anspach, L. C. Andreozzi, P. W. Kervin, and D. P. Dimiduk, "Advanced testing methods for acquisition, tracking, and pointing," in Orlando'90, 16-20 April, 1990, pp. 237-249.

[3] Y.-S. Kang, K. Moorhouse, and J. H. Bolte, Measurement of six degrees of freedom head kinematics in impact conditions employing six accelerometers and three angular rate sensors (6a $\omega$ configuration), J. Biomech. Eng-T. Asme. , Vol. 133, No. 11 (2011) 111007.

[4] G. W. Hall, "Measurement of elbow flexion and pronation with angular rate sensors," SAE Technical Paper, (1998).

[5] P. Martin, J. Crandall, W. Pilkey, C. Chou, and B. Fileta, "Measurement techniques for angular velocity and acceleration in an impact environment," Warrendale, PA, Rep.970575.(1997).

[6] A. Merkle, I. Wing, R. Szcepanowski, B. McGee, L. Voo, and M. Kleinberger, "Evaluation of angular rate sensor technologies for assessment of rear impact occupant responses," Baltimore ,USA, (2007).

[7] C. Pinney, M. A. Hawes, and J. Blackburn, "A cost-effective inertial motion sensor for short-duration autonomous navigation," in procceding of Position Location and Navigation Symposium, 1994, IEEE, 1994, pp. 591-597.

[8] D. Laughlin, H. Sebesta, and D. Eckelkamp-Baker, A dual function magnetohydrodynamic(MHD) device for angular motion measurement and control, Adv. Astronaut. Sci. , Vol. 111, No. (2002) 335-347.

[9] M. Xu, X. Li, T. Wu, C. Chen, and Y. Ji, Error Analysis of Theoretical Model of Angular Velocity Sensor Based on Magnetohydrodynamics at Low Frequency, Sensor. Actuat. A-Phys., Vol. 226, No. (2015) 116-125.

[10] O. Andreev, Y. Kolesnikov, and A. Thess, Experimental study of liquid metal channel flow under the influence of a nonuniform magnetic field, PHYS. FLUIDS., Vol. 18, No. 6 (2006) 065108. 